\section{Effect of HPV on cervical cancer screening in Alberta}

Kim and colleagues evaluated the dose-stratified effectiveness of the quadrivalent human papillomavirus (HPV) vaccine on reduction of cervical abnormalities in Alberta, using a nested case-control approach in data linked between the Alberta Health Care Insurance Plan and the Alberta provincial immunization repository. ${ }^{1}$ As expected, the authors found strong protection against high-grade cervical abnormalities among women who received three doses. The data appear to indicate similar protection conferred by one and two doses (odds ratio [OR], 0.45 and 0.17 , respectively), albeit with nonsignificant effect estimates, given the small case counts in these dose groups. Yet, the authors concluded that three doses were required for HPV vaccination to reduce highgrade cervical abnormalities. When we conducted an analysis of their results by combining women who received one or two HPV doses, significant protection against high-grade cervical abnormalities was seen (crude OR $0.29,95 \%$ confidence interval $[\mathrm{CI}]$ 0.09-0.93] when the controls were defined as normal cytology results).

We also have concerns about the study methods in Kim and colleagues' article. First, no HPV genotyping data were available (as is usually the case for these types of linkage studies). This is more problematic for outcomes such as low-grade squamous intraepithelial lesions and abnormal squamous cells of undetermined significance, where carcinogenic types other than HPV16/18 contribute to a great proportion of lesions. ${ }^{2,3}$ The data in their study indicated that vaccine effectiveness was greater with a high-grade endpoint, rather than with the less progressed endpoint(s).

Second, there was a lack of data about age at vaccination and covariates that might reflect prevaccination HPV exposure. Evaluation of age at first vaccination by dose group should have been possible because vaccination data were obtained from a provincial immunization repository. If reduced-dose recipients are systematically older or have an earlier age at sexual debut, they likely have more prevalent HPV infections at the time of vaccination that artificially lower the estimated effectiveness. A dosespecific analysis by time since vaccination, or application of a buffer period before case counting, could address this concern. Other published studies of post-licensure vaccine effectiveness by number of doses have had similar limitations. ${ }^{4,5}$

In conclusion, Kim and colleagues state that three doses were required for the vaccine to be effective against cervical abnormalities, thereby raising concerns about the effectiveness of reduced doses of HPV vaccination. We believe that this conclusion does not reflect the study data and that the inherent limitations in record linkage studies to address HPV vaccine effectiveness by dose were not adequately addressed. Further, the growing body of evidence from post-hoc analyses nested in trial settings continue to suggest that two doses (maybe even one) protect as well as three doses, at least in the short term. ${ }^{6,7}$

\section{Marc Brisson PhD}

Professor, Département de médecine sociale et preventive, Université Laval, Québec, Qué.; Canada Research Chair in Mathematical Modeling and Health Economics of Infectious Disease

\section{Mélanie Drolet PhD}

Senior research associate

Élodie Bénard, MSc

Research assistant, Axe Santé

des populations et pratiques optimales en santé, Centre de recherche du CHU de Québec-Université Laval, Québec, Qué.

\section{Aimée R. Kreimer PhD}

Senior investigator, Infections and Immunoepidemiology, National Cancer Institute, Rockville, MD

\section{References}

1. Kim J, Bell C, Sun M, et al. Effect of human papillomavirus vaccination on cervical cancer screening in Alberta. CMAJ 2016;188:E281-8.
2. Guan P, Howell-Jones R, Li N, et al. Human papillomavirus types in 115,789 HPV-positive women: a meta-analysis from cervical infection to cancer. Int J Cancer 2012;131:2349-59.

3. Clifford GM, Rana RK, Franceschi S, et al. Human papillomavirus genotype distribution in low-grade cervical lesions: comparison by geographic region and with cervical cancer. Cancer Epidemiol Biomarkers Prev 2005; 14:1157-64.

4. Herweijer E, Leval A, Ploner A, et al. Association of varying number of doses of quadrivalent human papillomavirus vaccine with incidence of condyloma. JAMA 2014;311:597-603.

5. Blomberg M, Dehlendorff C, Sand C, et al. Doserelated differences in effectiveness of human papillomavirus vaccination against genital warts: a nationwide study of 550,000 young girls. Clin Infect Dis 2015;61:676-82.

6. Kreimer AR, Rodriguez AC, Hildesheim A, et al Proof-of-principle evaluation of the efficacy of fewer than three doses of a bivalent HPV16/18 vaccine. J Natl Cancer Inst 2011;103:1444-51.

7. Kreimer AR, Struyf F, Del Rosario-Raymundo MR, et al. Efficacy of fewer than three doses of an HPV-16/18 AS04-adjuvanted vaccine: combined analysis of data from the Costa Rica Vaccine and PATRICIA Trials. Lancet Oncol 2015;16:775-86.

CMAJ 2016. DOI:10.1503/cmaj.1150121

\section{Functional impairment, not FASD}

The publication of new guidelines for fetal alcohol spectrum disorder (FASD) raises concerns about the ongoing push for FASD-specific assessment and support programs. ${ }^{1}$ Many children present with evidence of pervasive brain dysfunction who do not have FASD. Current developmental practice emphasizes interaction of function and environment, not etiologic labels. ${ }^{2}$

FASD is clinically indistinguishable from other causes of neurobehavioural disorders (e.g., antenatal alcohol or other teratogens, complex trauma, genetic). ${ }^{3}$ Treatment for neurobehavioural disorders is nonspecific (e.g., environmental supports) and based on function (e.g., cognitive, memory, executive, self-regulation). Emphasizing an etiologic label with no specific treatment is misleading to the public and unethical.

Function-based, nonmedical diagnosis-specific services are compatible with a vigorous public health program in preventing antenatal alcohol exposure. Basing resources on an etiologic label marginalizes individuals with neurobehavioural disabilities without a unifying diagnostic label. 